Original article

\title{
IS INTRAVENOUS LONG-ACTING OPIOID (MORPHINE) ASSOCIATED WITH MORE POSTOPERATIVE HYPOXEMIA THAN SHORT-ACTING OPIOID (FENTANYL) AFTER NON-CARDIAC SURGERY?
}

\author{
Ahmed Abdel Moniuom Ibrahium, Doaa Mohammed Farid, Lobna Taha El Dourgham, \\ Alshaimaa Abdel Fattah Kamel \\ Anaesthesia and Surgical Intensive Care Department, Faculty of Medicine, Zagazig University, \\ Zagazig, Egypt
}

Corresponding Author :

Alshaimaa Abdel Fattah

Kamel

Anaesthesia and Surgical

Intensive Care Department,

Faculty of Medicine, Zagazig

University, Zagazig, Egypt

waelmesallamy@gmail.com

$\begin{array}{ll}\text { Submit Date } & 2019-01-19 \\ \text { Revise Date } & 2019-03-14 \\ \text { Accept Date } & 2019-03-15\end{array}$

\section{ABSTRACT}

Background: morphine is a potent analgesic in decreasing postoperative pain; however its use is accompanied by many complications especially respiratory one. On the other side, fentanyl titration may cause less postoperative respiratory complications.

Objective: To compare between intravenous long-acting opioid (Morphine), and short-acting opioid (Fentanyl) regarding postoperative hypoxemia after non-cardiac surgery.

Patients and methods: A prospective randomized clinical study done on 52 patients, they were divided equally into two groups: Group M (Morphine) received $(0.1 \mathrm{mg} / \mathrm{kg}$ ), and Group F (Fentanyl) received (1 ug/ $/ \mathrm{kg}$ ) on induction, and supplemented by effective rescue analgesia. When postoperative VAS score $\geq 4$, patients in Group $\mathrm{M}$ received $5 \mathrm{mg}$ morphine as bolus ,and reassess pain every $5 \mathrm{~min}$ to give another bolus ( the total allowed dose was $15 \mathrm{mg} / 3-4 \mathrm{~h}$ ).For Group F 50ug fentanyl was given as a bolus , and could be repeated (total allowed dose was $100 \mathrm{ug} / 1-2 \mathrm{~h}$.).

Result(s): There were no significant difference between two groups as regard respiratory rate (RR) nor peripheral O2 saturation (SPO2) (P value $<0.05$ ), however there were significant higher $R R$ at $44 \mathrm{hrs}$ and $48 \mathrm{hrs}$ in fentanyl group, and higher SPO2 at $5 \mathrm{~min} ., 28 \mathrm{~h}$, and $40 \mathrm{~h}$ compared to morphine group. VAS score showed no significant difference between two groups, however fentanyl group show significant high VAS score at the first three hours. Nausea, vomiting, and itching show significant increase in morphine group compared to fentanyl group ( $\mathrm{P}$ value $>0.05$ ).

Conclusion: Intravenous morphine as effective rescue analgesic is not associated with more postoperative hypoxemia, or respiratory depression than fentanyl.

Keywords: opioid, morphine, fentanyl, and hypoxemia.

\section{INTRODUCTION}

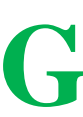
ood pain control following surgeries is important as it is associated with a decrease in the incidence of postoperative complications (1).

Intravenous rescue analgesia in the postoperative pain control is an effective method when perioperative multimodal analgesia fail to reduce it, titration of opioid analgesics in a patient suffering from severe, intolerable postoperative pain are effective method for controlling that pain (2).

Opioid have many side effects, the most serious one is respiratory depression due to suppression of respiratory center leading to hypoxemia (3).

Hypoxemia is defined as abnormally low level of oxygen in the arterial blood, and differs from hypoxia which means that the blood supplies less oxygen to the tissues (4). Hypoxemia is considered when oxygen saturation is less than $95 \%$ (5).

In an observational study done by Belcher ;et al in their study in 2016 on 40,000 patients found that no evidence that short- acting opioid (Fentanyl) decrease postoperative hypoxemia than long-acting opioid(Morphine) via patient controlled analgesia ( PCA) (6). 
The aim of this study was to compare between intravenous long-acting opioid (Morphine), and short-acting opioid (Fentanyl) on induction, and supplemented rescue analgesia regarding postoperative hypoxemia caused by these drugs in patients after non-cardiac surgery.

\section{PATIENTS AND METHODS}

The design for this prospective, randomized, clinical trial study was approved by our institutional ethics committee, and accordance with The Code of Ethics of the World Medical Association (Declaration of Helsinki) for studies involving humans. The written consent was obtained from all 52 patients after calculation of the sample size using Open Epi I program assuming that the mean of median area of postoperative oxyhemoglobin saturation under $95 \%$ in patients treated with short-acting opioid is $1.08 \pm 0.2$ versus $1.28 \pm 0.3$ in patients treated with longacting opioid (5) .The research was conducted between June 2017 and November 2017. Patients were included if they met the following criteria: scheduled for surgery under general anesthesia that exceed 48 hours postoperative hospital stay for non-cardiac surgery, age 21 to 45 years; American society of Anesthesiologists (ASA) classification I , II. The exclusion criteria were allergy to study drugs, pregnancy, respiratory disease, drug addiction ,liver, or kidney disease ,and mental retardation.

The mode of anesthesia was explained in details to all the patients. Patients were kept fasted for 8 hours before surgery. In the operation theater, electrocardiogram, pulse oximeter, and noninvasive blood pressure (NIBP) monitor were connected and baseline readings were obtained from each patient. In all patients, conventional general anesthesia technique was used. Intravenous (IV) Ringer lactate was started after securing an 18-G IV cannula at the rate of $250 \mathrm{~mL} / \mathrm{h}$. The patients were premedicated with IV ranitidine $50 \mathrm{mg}$, midazolam $0.05 \mathrm{mg} / \mathrm{kg}$, atropine $0.05 \mathrm{mg} / \mathrm{kg}$, in the theater. After preoxygenation with $100 \%$ oxygen for 3 minutes, general anaesthesia for the patients were induced with IV propofol $2 \mathrm{mg} / \mathrm{kg}$ and cis-atracurium $0.1 \mathrm{mg} / \mathrm{kg}$ and intubated with proper-sized cuffed endotracheal tube. In total, 52 patients were enrolled. Patients were computer generated randomly assigned to two groups. The groups were as follows: Group M: $(\mathrm{n}=$ 26) received morphine $0.1 \mathrm{mg} / \mathrm{kg}$, and Group F: $(\mathrm{n}=26)$ received fentanyl $1 \mathrm{ug} / \mathrm{kg}$.

anaesthesia was maintained with, 1.5MAC isoflurane and intermittent boluses of cisatracurium.

Extubation after fulfilling extubation criteria.

\section{Postoperative Data:}

After surgery, patients were transported to the recovery room, and basal vital signs of the patient were recorded: respiratory rate (RR), peripheral oxygen saturation (SPO2), heart rate and blood pressure just after recovery.

Pain score of the patient was recorded in the recovery room using Visual Analogue Scale (VAS score) from 0-10 ( 0 means no pain \& 10 means worst imaginable pain ).VAS was recorded every one hour for the first 4 hours then every 4 hours for the remaining time of the study"48 hours" (7).

* For Group M, If VAS score $=4$ or more, we gave morphine $5 \mathrm{mg}$ as a bolus and re-assess pain after $5 \mathrm{~min}$. If VAS score was still $\geq 4$, we could give a maximum of 3 boluses (total allowed dose $15 \mathrm{mg}$ every 3-4 hours).

* For Group F, If VAS score $\geq 4$, we gave fentanyl 50ug as a bolus and could be repeated after $5 \mathrm{~min}$ if still $\mathrm{VAS} \geq 4$, (the total allowed dose is 100 ug every 1-2 hours).

- Patients were observed in ward for 48 hours postoperatively for Postoperative hemodynamics, and the presence of adverse effects such as nausea, vomiting, sedation, and allergic reaction.

- We evaluated sedation score using $A=$ Alert. $\quad V=$ response To Verbal stimulus, $\mathrm{P}=$ response to Painful stimulus, $\mathrm{U}=$ Unresponsive (AVPU) (8).

- Also, patient satisfaction was assessed using 1-3 verbal scale. (1=well satisfied ,2 $=$ satisfied, $3=$ unsatisfied) (9).

- Any patient developed respiratory depression was treated as following:

1. Supplemental oxygen for patients with bradypnea (RR less than $10 \mathrm{~b} / \mathrm{m}$ ) or 
hypoxemia with $\mathrm{SpO} 2$ less than $95 \%$ till correction of hypoxemia. These cases will be recorded and continue with them till the end of the study. Although oxygen should be available during opioids administration, its continuous use may increase apneic episodes and alter sensitivity of pulse oximeter to detect hypoxemia (10).

2. If oxygen supply failed to correct respiratory depression naloxone should be considered and the case was recorded, admitted to ICU, treated, and excluded from the study. Recommended adult dose of naloxone for the treatment of severe opiate induced respiratory depression is 0.4 to $2 \mathrm{mg}$ intravenously every 2 to 3 minutes as necessary, simultaneously with assisted respiration. Maximum dose is $10 \mathrm{mg}$.

3. Ventilation also was considered.

- Also, any patient developed pain confirmed by VAS score $\geq 4$ after the maximum allowed dose of morphine (15mg) in group $\mathrm{M}$ or( $100 \mathrm{ug}$ ) fentanyl in group $\mathrm{F}$ was given non steroidal anti-inflammatory drugs ( diclofenac sodium $75 \mathrm{mg}$ amp. IM) and recorded.

- In case of vomiting, we administered 4 mg of ondansetron intravenously

- Constipation treated with laxatives as lactulose (dose: $15 \mathrm{ml}$ per dose orally until bowel movements return to normal) and enemas if needed.

\section{Data collected:}

- Patient's characteristics : age, sex, weight, ASA classification. Also vital signs: Systolic, and diastolic blood pressure, and heart rate (HR) were recorded.

- Type of the surgery.

- Respiratory rate (basal, 5min., 15min., 30min., 1h., 2h, 3h., 4h. then every 4 hours for 48hours).

- Peripheral oxygen saturation ( $\mathrm{SpO} 2)$ (basal, 5min., 15min., 30min., 1h., $2 \mathrm{~h} ., 3 \mathrm{~h}$. , $4 \mathrm{~h}$. then every 4 hours for 48hours).
- Pain score of the patient using VAS score every one hour for the first 4 hours then every 4 hours for 48 hours.

- Level of sedation using AVPU (alert, verbal, painful, unresponsive) score

- Patient satisfaction using 1-3 verbal scale (1=unsatisfactory analgesia, 2= satisfactory analgesia, $3=$ excellent satisfactory analgesia).

- Other complications related to study drugs: nausea, vomiting, constipation, or itching.

\section{Statistical analysis:}

At the end of study, all data were checked, entered and analyzed by using Special Package for Social Science) (SPSS) version 20. Data were expressed as mean \pm standard deviation for quantitative continuous variables, and number and percentage for categorical variables using chi-square $\left(\mathrm{X}^{2}\right)$.

$\mathrm{p}<0.05$ was considered statistically significant.

\section{RESULTS}

In this study, there were no significant differences regarding patient age, sex, weight, ASA physical status, and type of surgery between the two groups (table 1).

Regarding hemodynamics, our results showed no statistically significant difference of HR, SBP, and DBP between studied groups at the preset different times (table2).

In this study, fentanyl group showed significant higher RR at $44 \mathrm{hrs}$, and $48 \mathrm{hrs}$. as RR were $14.07 \pm 0.84$ and $14.26 \pm 0.72$ while in morphine group were $13.34 \pm 1.01$ and $13.73 \pm 0.87$ respectively ( $\mathrm{p}$ value $<0.05$ ) otherwise no significant difference between the studied groups (table 3).

Also, SPO2 was significantly higher in fentanyl group at 5 minutes, 28 hours and 40 hours as $\mathrm{SpO} 2$ were $96.2 \pm 0.71,95.22 \pm 0.93$ and $95.34 \pm 0.93$ respectively while in morphine group were $95.26 \pm 0.77,94.19 \pm 1.09$ and $94.8 \pm 1.15$ respectively ( $\mathrm{p}$ value $<0.05$ ) otherwise no significant difference between the two studied groups ( $\mathrm{p}$ value $>0.05)$ (table4)

In this study, fentanyl group showed significant higher VAS score at $1 \mathrm{~h}, 2 \mathrm{~h}$, and $3 \mathrm{~h}$. as VAS were $5.69 \pm 0.47,4.61 \pm 0.63$, and $3.9 \pm 0.81$ respectively, while in morphine group VAS score were $4.5 \pm 1.6,3.2 \pm 0.82$, 310 
Ahmed et al..

Zagazig University Medical Journals

and 3.2 \pm 1.1 respectively otherwise no significant difference between studied groups (p value >0.05) (Fig. 1)(table 5).

In this study, Morphine group show significantly higher incidence of nausea, vomiting and itching as the percent were $61.5 \%, 53.8 \%$ and $73.1 \%$ respectively while in fentanyl group percentage were $34.6 \%$,
$26.9 \%$ and $34.6 \%$ respectively ( $\mathrm{p}$ value $<0.05$ ) but there was no significant difference regarding constipation between the two study groups ( $\mathrm{p}$ value $>0.05)$ (table 6).

Regarding level of sedation, and degree of patient satisfaction, there were no significant difference between both groups ( $\mathrm{p}$ value $<0.05)$ (table 7).

Table 1 Patients data and type of surgery of the studied groups.

\begin{tabular}{|l|c|c|c|c|}
\hline \multicolumn{1}{|c|}{ Variables } & $\begin{array}{c}\text { Morphine group } \\
(\mathrm{n}=26)\end{array}$ & $\begin{array}{c}\text { Fentanyl group } \\
(\mathrm{n}=26)\end{array}$ & t- value & p- value \\
\hline Age (years) & $37.23 \pm 6.38$ & $34.46 \pm 6.54$ & 1.54 & $0.13(\mathrm{NS})$ \\
$\overline{\mathrm{X}}_{ \pm \text {SD }}$ & & & & \\
\hline Weight (kg) & $85.92 \pm 7.75$ & $85.34 \pm 9.57$ & 0.24 & $0.81(\mathrm{NS})$ \\
$\overline{\mathrm{X}}_{ \pm \text {SD }}$ & $\mathrm{N}(\%)$ & $\mathrm{N}(\%)$ & $\chi^{2}=0.08$ & $0.77(\mathrm{NS})$ \\
\hline Sex & $11(42.3)$ & $12(46.2)$ & & \\
Male & $15(57.7)$ & $14(53.8)$ & & \\
Female & $20(76.9)$ & $22(84.6)$ & $\chi^{2}=0.57$ & $0.75(\mathrm{NS})$ \\
\hline ASA & $6(23.1)$ & $4(15.4)$ & & \\
I & & & & \\
II & $17(32.6 \%)$ & $18(34.6 \%)$ & & \\
\hline Type of surgery & $20(38.5 \%)$ & $16(30.7 \%)$ & $\chi^{2}=0.07$ & $0.76(\mathrm{NS})$ \\
Maxillofacial & $15(28.8 \%)$ & $18(34.6 \%)$ & & \\
Abdominal & & &
\end{tabular}

$\chi^{2}$ chi square test $\quad \mathrm{NS}=$ non-significant

Data were expressed as mean $\pm \mathrm{SD}$, or number (percentage).

Table 2 Comparison of Heart rate (HR) (beat per minute), systolic blood pressure (SBP), and diastolic blood pressure (DBP) (mmHg) distribution between morphine and fentanyl groups at different times.

\begin{tabular}{|l|l|l|l|l|}
\hline $\begin{array}{l}\text { Time of } \\
\text { measurement }(\mathbf{H R})\end{array}$ & Morphine(26) & Fentanyl(26) & $\mathbf{t}$ & $\mathbf{p}$ \\
\hline At basal & $96.57 \pm 4.77$ & $92.96 \pm 3.28$ & 3.182 & 0.003 \\
\hline At $1 \mathrm{~h}$ & $90.88 \pm 6.16$ & $88.0 \pm 3.57$ & 2.063 & 0.044 \\
\hline At $2 \mathrm{~h}$ & $87.19 \pm 7.03$ & $83.96 \pm 4.11$ & 2.021 & 0.049 \\
\hline At 3h & $86.20 \pm 6.03$ & $82.97 \pm 4.11$ & 2.020 & 0.048 \\
\hline At 4h & $85.69 \pm 8.01$ & $81.73 \pm 3.43$ & 2.317 & 0.025 \\
\hline At $8 \mathrm{~h}$ & $83.5 \pm 7.57$ & $81.0 \pm 5.28$ & 1.380 & 0.174 \\
\hline At $12 \mathrm{~h}$ & $82.57 \pm 6.91$ & $80.03 \pm 5.48$ & 1.466 & 0.149 \\
\hline At $18 \mathrm{~h}$ & $81.0 \pm 5.74$ & $79.11 \pm 5.63$ & 1.194 & 0.238 \\
\hline At 24h & $82.42 \pm 6.5$ & $80.46 \pm 6.26$ & 1.108 & 0.273 \\
\hline At 30h & $81.73 \pm 6.29$ & $78.76 \pm 5.92$ & 1.748 & 0.087 \\
\hline At 36h & $81.0 \pm 5.74$ & $79.11 \pm 5.63$ & 1.194 & 0.238 \\
\hline At 42h & $80.26 \pm 5.76$ & $78.92 \pm 5.48$ & 0.863 & 0.392 \\
\hline At 44h & $80.25 \pm 5.76$ & $78.91 \pm 5.48$ & 0.862 & 0.391 \\
\hline At 48h & $80.15 \pm 5.72$ & $79.15 \pm 5.46$ & 0.645 & 0.522 \\
\hline SBP(mmHg) & Morphine (26) & Fentanyl (26) & T & p \\
\hline At basal & $134.42 \pm 7.78$ & $135.76 \pm 8.9$ & -0.580 & 0.564 \\
\hline At $1 \mathrm{~h}$ & $130.38 \pm 6.77$ & $130.0 \pm 8.0$ & 0.187 & 0.852 \\
\hline At 2h & $129.03 \pm 7.74$ & $126.34 \pm 7.94$ & 1.237 & 0.222 \\
\hline At 3h & $129.02 \pm 7.74$ & $125.34 \pm 7.94$ & 1.236 & 0.221 \\
\hline
\end{tabular}


Ahmed et al..

Continued Table 2

\begin{tabular}{|l|l|l|l|l|}
\hline $\begin{array}{l}\text { Time of } \\
\text { measurement (HR) }\end{array}$ & Morphine(26) & Fentanyl(26) & $\mathbf{t}$ & p \\
\hline & & & & \\
\hline At 4h & $125.5 \pm 9.61$ & $124.03 \pm 6.32$ & 0.647 & 0.520 \\
\hline At 8h & $123.26 \pm 7.34$ & $122.69 \pm 6.36$ & 0.303 & 0.763 \\
\hline At 12h & $121.73 \pm 6.31$ & $121.73 \pm 6.31$ & 0.002 & 0.999 \\
\hline At 18h & $122.3 \pm 7.9$ & $119.80 \pm 4.99$ & 1.363 & 0.179 \\
\hline At 24h & $122.88 \pm 5.68$ & $120.19 \pm 5.56$ & 1.725 & 0.091 \\
\hline At 30h & $122.3 \pm 7.9$ & $119.80 \pm 4.99$ & 1.363 & 0.179 \\
\hline At 36h & $120.38 \pm 5.27$ & $119.61 \pm 5.98$ & 0.491 & 0.625 \\
\hline At 42h & $120.19 \pm 6.24$ & $120.96 \pm 6.16$ & 0.447 & 0.657 \\
\hline At 44h & $120.20 \pm 6.24$ & $121.96 \pm 6.16$ & 0.447 & 0.657 \\
\hline At 48h & $120.96 \pm 6.0$ & $122.11 \pm 6.5$ & 0.665 & 0.509 \\
\hline DBP(mmHg) & Morphine (26) & Fentanyl (26) & $\mathbf{t}$ & $\mathbf{p}$ \\
\hline At basal & $83.38 \pm 7.28$ & $85.38 \pm 9.04$ & 0.878 & 0.384 \\
\hline At 1h & $80.57 \pm 7.25$ & $79.23 \pm 6.88$ & 0.686 & 0.496 \\
\hline At 2h & $76.92 \pm 6.01$ & $78.07 \pm 7.49$ & 0.612 & 0.543 \\
\hline At 3h & $76.91 \pm 6.01$ & $78.06 \pm 7.49$ & 0.613 & 0.542 \\
\hline At 4h & $76.53 \pm 7.97$ & $74.42 \pm 8.98$ & 0.898 & 0.373 \\
\hline At 8h & $75.38 \pm 5.27$ & $75.76 \pm 4.83$ & 0.274 & 0.785 \\
\hline At 12h & $76.92 \pm 4.7$ & $76.15 \pm 4.96$ & 0.574 & 0.569 \\
\hline At 18h & $76.03 \pm 5.37$ & $73.53 \pm 12.34$ & 0.947 & 0.348 \\
\hline At 24h & $76.15 \pm 6.37$ & $72.57 \pm 12.22$ & 1.323 & 0.192 \\
\hline At 30h & $76.03 \pm 5.37$ & $73.53 \pm 12.34$ & 0.947 & 0.348 \\
\hline At 36h & $76.53 \pm 4.64$ & $76.15 \pm 4.96$ & 0.289 & 0.774 \\
\hline At 42h & $75.57 \pm 5.71$ & $75.0 \pm 5.09$ & 0.384 & 0.703 \\
\hline At 44h & $75.55 \pm 5.7$ & $76.1 \pm 5.09$ & 0.383 & 0.702 \\
\hline At 48h & $75.57 \pm 6.37$ & $76.73 \pm 5.46$ & 0.701 & 0.487 \\
\hline Data we & & \\
\hline
\end{tabular}

Data were expressed as mean $\pm \mathrm{SD}$, or number (percentage).

Table 3 Comparison of respiratory rate (RR) (breath per minute) between morphine and fentanyl groups at different times.

\begin{tabular}{|l|c|c|c|c|}
\hline Time of measurement & $\begin{array}{c}\text { Morphine group (n=26) } \\
\text { mean } \pm \text { SD }\end{array}$ & $\begin{array}{c}\text { Fentanyl group (n=26) } \\
\text { mean } \pm \text { SD }\end{array}$ & $\mathbf{t}$ \\
\hline Basal RR (BPM) & $18.61 \pm 1.06$ & $18.84 \pm 1.31$ & 0.7 & 0.49 \\
\hline At 5min. & $16.3 \pm 1.22$ & $16.8 \pm 1.13$ & 1.53 & 0.13 \\
\hline At 15min. & $14.61 \pm 1.26$ & $14.88 \pm 1.1$ & 0.82 & 0.42 \\
\hline At 30min. & $13.69 \pm 1.22$ & $13.15 \pm 1.18$ & 1.61 & 0.11 \\
\hline At 1h. & $13.15 \pm 1.54$ & $13.34 \pm 1.29$ & 0.49 & 0.63 \\
\hline At 2h & $13.57 \pm 1.17$ & $13.84 \pm 1.22$ & 0.81 & 0.42 \\
\hline At 3h & $13.89 \pm 1.32$ & $13.5 \pm 1.13$ & 1.44 & 0.26 \\
\hline At 4h & $13.84 \pm 1.28$ & $14.0 \pm 1.16$ & 0.45 & 0.65 \\
\hline At $8 \mathrm{~h}$ & $13.65 \pm 1.23$ & $13.88 \pm 1.03$ & 0.73 & 0.47 \\
\hline At $12 \mathrm{~h}$ & $13.61 \pm 1.47$ & $13.3 \pm 1.25$ & 0.81 & 0.42 \\
\hline At $16 \mathrm{~h}$ & $13.61 \pm 1.06$ & $13.92 \pm 1.19$ & 0.98 & 0.33 \\
\hline At 20h & $14.03 \pm 1.03$ & $13.92 \pm 0.89$ & 0.43 & 0.67 \\
\hline At 24h & $13.65 \pm 1.32$ & $13.8 \pm 0.74$ & 0.52 & 0.61 \\
\hline At 28h & $13.73 \pm 1.0$ & $14.0 \pm 1.16$ & 0.89 & 0.38 \\
\hline At 32h & $13.73 \pm 1.31$ & $13.96 \pm 0.82$ & 0.76 & 0.45 \\
\hline At 36h & $13.8 \pm 0.93$ & $13.88 \pm 1.17$ & 0.26 & 0.8 \\
\hline At 40h & $13.84 \pm 0.92$ & $14.03 \pm 0.87$ & 0.77 & 0.44 \\
\hline At 44h & $13.34 \pm 1.01$ & $14.07 \pm 0.84$ & 2.82 & $0.007^{*}$ \\
\hline At 48h & $13.73 \pm 0.87$ & $14.26 \pm 0.72$ & 2.42 & $0.019^{*}$ \\
\hline
\end{tabular}

*significant $(\mathrm{p}<0.05)$

Data were expressed as mean $\pm \mathrm{SD}$ 
Table 4 Comparison of peripheral oxygen saturation (SPO2) between morphine and fentanyl groups at different times.

\begin{tabular}{|l|c|c|c|c|}
\hline $\begin{array}{l}\text { Time of } \\
\text { measurement }\end{array}$ & $\begin{array}{c}\text { Morphine group } \\
(\mathbf{n = 2 6 )} \\
\text { mean } \pm \text { SD }\end{array}$ & $\begin{array}{c}\text { Fentanyl group } \\
(\mathbf{n = 2 6 )} \\
\text { mean } \pm \text { SD }\end{array}$ & $\mathbf{t}$ & $\mathbf{p}$ \\
\hline At basal & $96.23 \pm 0.81$ & $96.3 \pm 0.61$ & 0.38 & 0.7 \\
\hline At 5 min. & $95.26 \pm 0.77$ & $96.23 \pm 0.71$ & 2.79 & $0.002^{*}$ \\
\hline At 15min. & $94.23 \pm 1.03$ & $94.73 \pm 1.02$ & 1.18 & 0.21 \\
\hline At 30min. & $94.69 \pm 1.31$ & $94.73 \pm 1.21$ & 0.11 & 0.91 \\
\hline At 1h. & $94.92 \pm 0.84$ & $94.69 \pm 1.15$ & 0.82 & 0.42 \\
\hline At 2h & $94.76 \pm 0.9$ & $94.8 \pm 1.02$ & 0.14 & 0.89 \\
\hline At 3h & $94.32 \pm 1.41$ & $94.16 \pm 0.83$ & 0.49 & 0.62 \\
\hline At 4h & $94.78 \pm 0.89$ & $94.48 \pm 0.94$ & 1.12 & 0.21 \\
\hline At 8h & $94.61 \pm 1.02$ & $94.46 \pm 1.02$ & 0.54 & 0.59 \\
\hline At 12h & $94.53 \pm 1.52$ & $94.53 \pm 1.06$ & 0.003 & 0.99 \\
\hline At 16h & $94.53 \pm 1.1$ & $94.61 \pm 0.98$ & 0.27 & 0.79 \\
\hline At 20h & $94.23 \pm 1.33$ & $94.3 \pm 1.12$ & 0.23 & 0.82 \\
\hline At 24h & $94.19 \pm 1.2$ & $94.69 \pm 0.67$ & 1.85 & 0.07 \\
\hline At 28h & $94.19 \pm 1.09$ & $95.22 \pm 0.93$ & 2.59 & $0.013^{*}$ \\
\hline At 32h & $92.61 \pm 9.57$ & $94.8 \pm 0.93$ & 1.16 & 0.25 \\
\hline At 36h & $94.65 \pm 1.09$ & $94.84 \pm 0.92$ & 0.69 & 0.5 \\
\hline At 40h & $94.73 \pm 1.15$ & $95.34 \pm 0.93$ & 2.1 & $0.039^{*}$ \\
\hline At 44h & $94.96 \pm 0.91$ & $94.96 \pm 0.72$ & 0.002 & 0.99 \\
\hline At 48h & $95.5 \pm 0.81$ & $95.38 \pm 1.02$ & 0.45 & 0.65 \\
\hline
\end{tabular}

*significant $(\mathrm{p}<0.05)$.

Data were expressed as mean $\pm \mathrm{SD}$

Table 5 Comparison of Visual Analogue Scale (VAS) score between morphine and fentanyl groups at different times.

\begin{tabular}{|l|c|c|c|c|}
\hline $\begin{array}{l}\text { Time of } \\
\text { measurement }\end{array}$ & $\begin{array}{c}\text { Morphine group } \\
(\mathbf{n = 2 6 )} \\
\text { mean } \pm \text { SD }\end{array}$ & $\begin{array}{c}\text { Fentanyl group } \\
(\mathbf{n = 2 6 )} \\
\text { mean } \pm \text { SD }\end{array}$ & $\mathbf{t}$ & $\mathbf{p}$ \\
\hline At basal & $7.2 \pm 0.48$ & $7.0 \pm 0.44$ & 1.5 & 0.12 \\
\hline At $1 \mathrm{~h}$ & $4.5 \pm 1.6$ & $5.69 \pm 0.47^{*}$ & 3.6 & 0.001 \\
\hline At $2 \mathrm{~h}$ & $4.03 \pm 0.82$ & $4.61 \pm 0.63^{*}$ & 2.8 & 0.006 \\
\hline At 3h & $3.2 \pm 1.1$ & $3.9 \pm 0.81^{*}$ & 2.6 & 0.01 \\
\hline At 4h & $3.76 \pm 1.21$ & $3.73 \pm 0.45$ & 0.15 & 0.88 \\
\hline At $8 \mathrm{~h}$ & $3.34 \pm 1.01$ & $3.0 \pm 0.63$ & 1.47 & 0.15 \\
\hline At $12 \mathrm{~h}$ & $3.42 \pm 1.33$ & $3.23 \pm 0.95$ & 0.56 & 0.55 \\
\hline At $16 \mathrm{~h}$ & $3.0 \pm 1.09$ & $3.34 \pm 0.97$ & 1.2 & 0.24 \\
\hline At $20 \mathrm{~h}$ & $3.07 \pm 0.97$ & $3.46 \pm 0.94$ & 1.4 & 0.16 \\
\hline At $24 \mathrm{~h}$ & $2.76 \pm 1.21$ & $2.88 \pm 0.71$ & 0.42 & 0.68 \\
\hline At 28h & $2.88 \pm 1.07$ & $2.61 \pm 0.69$ & 1.1 & 0.29 \\
\hline At 32h & $2.61 \pm 1.23$ & $2.38 \pm 0.69$ & 0.83 & 0.4 \\
\hline At 36h & $2.69 \pm 1.22$ & $2.3 \pm 0.59$ & 1.46 & 0.39 \\
\hline At 40h & $2.1 \pm 0.84$ & $2 \pm 0.8$ & 0.44 & 0.66 \\
\hline At 44h & $2.03 \pm 0.91$ & $2.1 \pm 0.64$ & 0.32 & 0.74 \\
\hline At 48h & $1.7 \pm 0.74$ & $1.46 \pm 0.58$ & 1.3 & 0.2 \\
\hline
\end{tabular}

*significant $(\mathrm{p}<0.05)$.

Data were expressed as mean $\pm \mathrm{SD}$ 
Table 6 Post anesthesia complications of morphine, and fentanyl groups:

\begin{tabular}{|c|c|c|c|c|}
\hline Variables & $\begin{array}{l}\text { Morphine group } \\
(\mathrm{n}=26) \\
\mathrm{N}(\%)\end{array}$ & $\begin{array}{l}\text { Fentanyl group } \\
(\mathrm{n}=26) \\
\mathrm{N}(\%)\end{array}$ & $\chi^{2}$ & $\mathrm{p}$ - value \\
\hline nausea & $16(61.5)$ & $9(34.6)$ & 3.8 & $0.026(\mathrm{~S})$ \\
\hline Vomiting & $14(53.8)$ & $7(26.9)$ & 3.1 & $0.038(\mathrm{~S})$ \\
\hline Itching & $19(73.1)$ & $9(34.6)$ & 7.7 & $0.005(\mathrm{~S})$ \\
\hline Constipation & $12(46.2)$ & $7(26.9)$ & 2.07 & $0.15(\mathrm{NS})$ \\
\hline
\end{tabular}

Table 7 Post anesthesia sedation and satisfaction of morphine, and fentanyl groups:

\begin{tabular}{|l|c|c|c|c|}
\hline \multicolumn{1}{|c|}{ Variables } & $\begin{array}{c}\text { Morphine group } \\
(\mathrm{n}=26) \\
\mathrm{N}(\%)\end{array}$ & $\begin{array}{c}\text { Fentanyl group } \\
(\mathrm{n}=26) \\
\mathrm{N}(\%)\end{array}$ & $\chi^{2}$ & $\mathrm{p}$ - value \\
\hline Sedation level (AVPU score ) & $21(80.7)$ & $23(88.4)$ & & \\
Alert & $3(11.6)$ & $2(7.7)$ & 0.62 & $0.73(\mathrm{NS})$ \\
Verbal & $2(7.7)$ & $1(3.9)$ & & \\
Pain & 0 & 0 & & \\
Un-response & $20(76.9)$ & $19(73.1)$ & 0.22 & $0.89(\mathrm{NS})$ \\
\hline Satisfaction (1-3verbal scale) & $4(15.4)$ & $4(15.4)$ & & \\
excellent satisfied & $2(7.7)$ & $3(11.5)$ & & \\
satisfied & & & & \\
un-satisfied & &
\end{tabular}

$\chi^{2}$ chi square test $\quad \mathrm{NS}=$ non-significant $\mathrm{N}=$ number

Data were expressed as number (percentage)

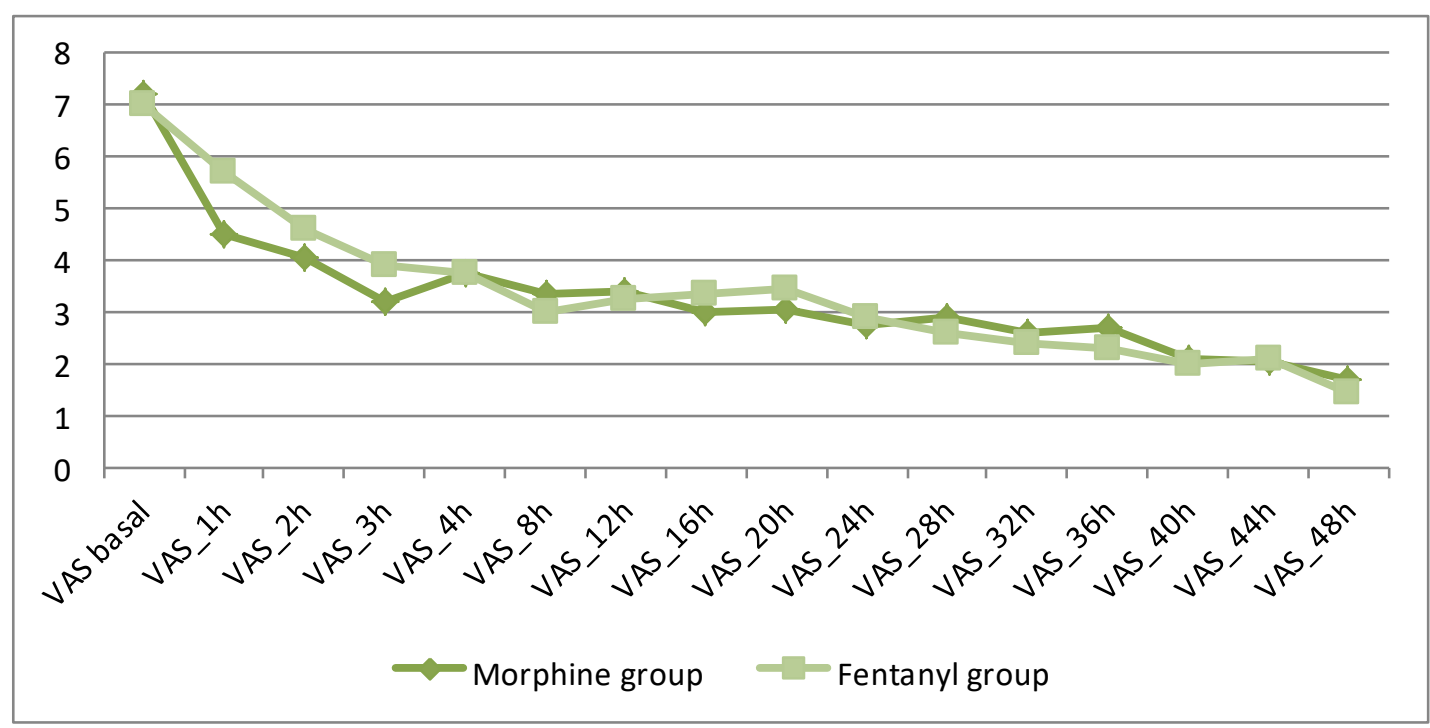

Figure1 Visual Analogue Scale (VAS) score of morphine and fentanyl group at different times.

\section{DISCUSSION}

Many anesthesiologists believe that long-acting opioids provoke more hypoxemia relative to short-acting opioids, which is consistent with the observation that longacting opioids produce more sedation than short-acting opioids. Nonetheless, the amount of ward hypoxemia was similar for longacting and short-acting opioids given through IV administration in adults recovering from non-cardiac surgery (4). 
Opioids are widely used as analgesics and this effect is mediated via opiate receptors in nervous system leading to suppression of pain signals (11).

In this study we compare the effect of long-acting opioid (morphine) and the shortacting opioid (fentanyl) as postoperative analgesia regarding degree of postoperative hypoxemia produced by them, and other complications related to these drugs

Regarding postoperative hypoxemia and respiratory depression, our results agree with Allan et al (5) who said that long acting opioid morphine is not associated with more postoperative hypoxemia than short acting opioid fentanyl after non cardiac surgery denoting that no significant difference between the two groups, and with Adriana et al (2) who concluded that morphine, and fentanyl are equally effective in the Postoperative pain management with no significant respiratory depression.

Our results revealed that no significant changes in respiratory rate between the two groups except at 44hs., and 48hs.where patients in morphine group were associated with lower RR than patients in fentanyl group but this lowering did not reach level of respiratory depression ( $R R<8$ breath per minute) and this was in agreement with Allan et al (5) who said that morphine is not associated with more postoperative respiratory depression than fentanyl after non cardiac surgery.

Regarding VAS score, our results showed that there was no significant difference between the two study groups and this was in agreement with Adriana et al (2), and Andrea et al (12) who concluded that there were no significant difference in VAS score between morphine and fentanyl denoting that they are equally effective in treating postoperative pain .

Our results revealed that VAS score was higher in fentanyl group readings 1, 2, and 3 hours as fentanyl is short acting opioid and its duration of action is $30-60$ minutes after initial bolus due to redistribution then duration increased with repeated boluses as in Refika et al (13), and El Aish etal (14).

Regarding nausea, vomiting and itching, our results are in agreement with El Aish et al (14) who revealed that morphine is associated with more postoperative nausea, vomiting and itching than fentanyl and other opioids through their action on $\mathrm{Mu}$ receptors on chemoreceptor trigger zone (CTZ) and on myenteric, and submucosal plexi leading to delayed gastric emptying .

Regarding constipation, our results showed no significant difference between the two studied groups and this is agreement with Smith et al (15).

Regarding level of sedation, and degree of satisfaction, there were no significant difference between the two studied groups and in agreement with Adriana et al (7)

\section{CONCLUSION}

From the results of our study, we can conclude that although intravenous morphine showing potent pharmacological effect than fentanyl, there were no significant respiratory depression or hypoxemia lasting for $48 \mathrm{hrs}$ postoperative in non- cardiac surgery, Also both of them showing effective supplemented rescue analgesia provided that they are given at the prescribed doses as in our study.

\section{Declaration of interest}

The authors report no conflicts of interest.

The authors alone are responsible for the content and writing of the paper.

\section{Funding information}

None declared

\section{REFERENCES}

1. Ranguis SC, Li D, Webster AC,( 2010): Perioperative epidural steroids for lumbar spine surgery in degenerative spinal disease: a review. J Neurosurg Spine. 13:745-757

2. Adriana CP, Francisco JB, Olga GS et al. (2017): Comparison of the effectiveness of fentanyl versus morphine for severe postoperative pain management. A randomized, double blind, clinical trial; Colombian Journal of Anesthesiology. 45: 100-107

3. . Sun Z, Sessler DI, Dalton JE et al. (2015) : Postoperative hypoxemia is common and persistent: a prospective blinded observational study. AnesthAnalg.121:709-715.

4. Butterworth JF, Mackey DC and Wasnick JD. (2013) : Respiratory Physiology \& Anesthesia. Morgan \& Mikhail's Clinical Anesthesiology. 5th ed. New York, NY: McGraw-Hill :487-526. 
Ahmed et al..

5. Allan WB, Ashish KK, Steve $L$ et al. (2016): long-acting opioids are not associated with more postoperative hypoxemia than short-acting opioids after non cardiac surgery: AnesthAnalg. ;123 :1471-1479.

6. Belcher AW, Khanna AK, Leung S, et al. (2016):Long-acting patient-controlled opioids are not associated with more postoperative hypoxemia than shortacting patient-controlled opioids after noncardiac surgery:a cohort analysis.Anesth Analg. in press.

7. Gillian A, Samra Mian, Tetyana Kendzerska et al. (2011): Measures of adult pain: Visual Analog Scale for pain(VAS Pain), Numeric Rating Scale for pain (NAS Pain), McGill Pain Questionnaire (MPQ), Questionnaire (SFMPQ), Chronic Pain Grade Scale (CPGS), Short Form - 36 Bodily Pain Scale (SF - 36 BPS), and Measure of Intermittent and Constant Osteoarthritis Pain (ICOAP).Arthritis Care \& Research,63,S11.

8. Kelly AC, Upex A and Bateman DN. (2005): Comparison of consciousness level assessment in the poisoned patient using the alert/verbal/painful/unresponsive scale and the Glascow coma scale. Annals of Emergency Medicine; 44(2):108-113.

9. Ross VA, Pan PH, Medge D, et al. (2009): Neostigmine decreases

\section{Zagazig University Medical Journals}

bupivacaine use by patient-controlled epidural analgesia during labor: A randomized controlled study. Anesth Analg.109 (2):564-531.

10. Carvalho B. (2008): Respiratory depression after neuraxial opioids in the obstetric setting. Anesthesia \& Analgesia; 107(3): 956-961.

11. Benzon, Honorio, Raja et al. (2011): Essentials of Pain Medicine. Elsevier HealthSciences.: 85.

12. Andrea Russo, Domenico Luca Grieco, Francesca Bevilacqua et al (2017): Continunous intravenous analgesia with fentanyl or morphine after gynecological surgery:a cohort study. Journal of Anesthesia Vol.(31),issue(1),pp 51-57.

13. Refika Kilickaya, Yavuz Orak,Mehtap Arda et a.(2016):Comparison of the effect of intrathecal fentanyl and intrathecal morphine on pain in elective total knee replacement surgery.Journal of pain research and management Vol.2016.

14. EL Aish KIA, Tafish R,and Zourob.(2018):Morphine versus Fentanyl Used Spinally for post Cesarean Section Analgesia: A Randomized Clinical Trail.Journal of Anesthesia and Therapeutic.Vol.(1),issue(1).

15. Smith MD, Wang Y, Cudnik $M$ et al.(2012): The effectiveness and adverse event of morphine versus fentanyl on a physician helicopter.J Emerg Med. Jul;43(1):69-75.

To cite this article: Ibrahium AA,Farid DM $\mathrm{DM}_{L}$ El Dourgham LT,Kamel AA. IS Intravenous Long-Acting Opioid (Morphine) Associated with More Postoperative Hypoxemia Than Short-Acting Opioid (Fentanyl) After NonCardiac Surgery?,Egypt.ZUMJ 2019;25(3);308-316,DOI: 10.21608/zumj.2019.7367.1028 